\title{
Correction of Anterior Vertical Dusplasia by using Tongue Crib Therapy in An Adult Patient -A Cose Report
}

\author{
Dr Kratika Mishra,' Dr Amit Bhardwaj² \\ 'Assistant Professor, '2Dean, Professor and Head, Department of Orthodontics and Dentofacial Orthopaedics, \\ Modern Dental College and Research Centre, Indore, India
}

Correspondence: Dr Kratika Mishra; Email: kratikamishra19go@gmail.com

\section{ABSTRACT}

\begin{abstract}
A female patient of 26 years reported to the Department of Orthodontics, with a chief complaint of spacing between upper and lower front teeth. Patient has a habit of thumb sucking during night time since last 24 years. On extra oral examination she has mesoprosopic facial form, competent lips, bilaterally asymmetric face, convex profile, acute nasolabial angle, high clinical FMA. Intra oral examination reveals anterior open bite with Angle's Class I molar relation and class I canine relation, proclined upper and lower anterior teeth slightly crowded. Cephalometrically skeletal class II malocclusion, vertical growth pattern with bidental proclination with $10 \mathrm{~mm}$ openbite. Detailing treatment strategies such as bracket repositioning, occlusal adjustment, and elastics were used.
\end{abstract}

Keywords: Habit breaking appliance, Open bite, Vertical control

\section{INTRODUCTION}

Achieving an ideal treatment outcome depends on an accurate diagnosis in three dimensions, a good understanding of the interaction between the neuromuscular components of the orofacial region and the craniofacial skeleton, and the ability to provide individualised treatment mechanics.' There is mutual dependence in three dimensions of the Dentofacial complex according to early research in 1950's and 1960's. ${ }^{2}$

The importance of vertical dimension of the face was first recognised by Downs and later discussed by Wylie and Johnson. ${ }^{3}$ Sassouni and Nanda ${ }^{2}$ comprehensively described the skeletal characteristics caused by dimensional and positional imbalances in both vertical and anteroposterior dimensions. Their classification of facial types included an assessment of vertical disproportions. The terms skeletal deep bite and skeletal open bite were used to describe the two extremes observed in this dimension. ${ }^{2,4}$

Open bite has been defined as an occlusal characteristic in which upper and lower teeth are not in contact and vertical overlap does not exist. The term was first introduced by Caravelli as early as $1842 .{ }^{5}$ The anterior open bite remains one of the most challenging cases to treat in orthodontics. ${ }^{6}$ The prevalence of an anterior open bite varies with age and among ethnic groups and ranges from 1 to $11.5 \% .^{7-9}$ The aetiology of anterior open bite may be multifactorial; it often requires comprehensive, multidisciplinary treatment. Physical characteristics such as facial pattern may be involved in its development; 10 other contributing factors include habits such as non-nutritive sucking, ${ }^{11,12}$ abnormal swallowing, atypical speech, mouth breathing, variable resting tongue postures, ${ }^{13}$ and occlusive and eruptive forces in disequilibrium. ${ }^{14}$ In cases involving non-nutritive sucking habits, orthodontic appliances that promote habit suspension and tongue repositioning have been shown to be more effective than no treatment. ${ }^{15,16}$ Thumb Sucking and hyper divergent facial pattern are significant risk factors not only for development but also increased severity of an open bite.

\section{CASE REPORT}

A 26 year old female patient presented in the department of orthodontics with the chief complaint of spacing between upper and lower front teeth. The pre-treatment extra-oral (Fig. 1), intra-oral photographs (Fig. 2) and cephalogram and a panoramic radiograph (Fig. 3) were taken. On Extraoral examination she had mesoprosopic facial form, competent lips, bilaterally 
asymmetrical face, convex profile, acute nasolabial angle, high clinical FMA. On Intraoral examination presence of anterior open bite of -10mm was observed. In addition, two distinct occlusal planes were present in the upper arch with Angle's Class I molar and class I canine relation with proclined upper and lower anterior teeth. Cephalometrically, (Table 1) skeletal class II malocclusion ANB (6 degrees) with mandible retro positioned ( $N$ prep Pog -9), vertical growth pattern (FMA - 35 degrees) with bidental proclination and decreased anterior and posterior dental height. Soft tissue profile reveals protruded upper and lower lip. Patient had a habit of thumb sucking since last 24 years during night time unconsciously.
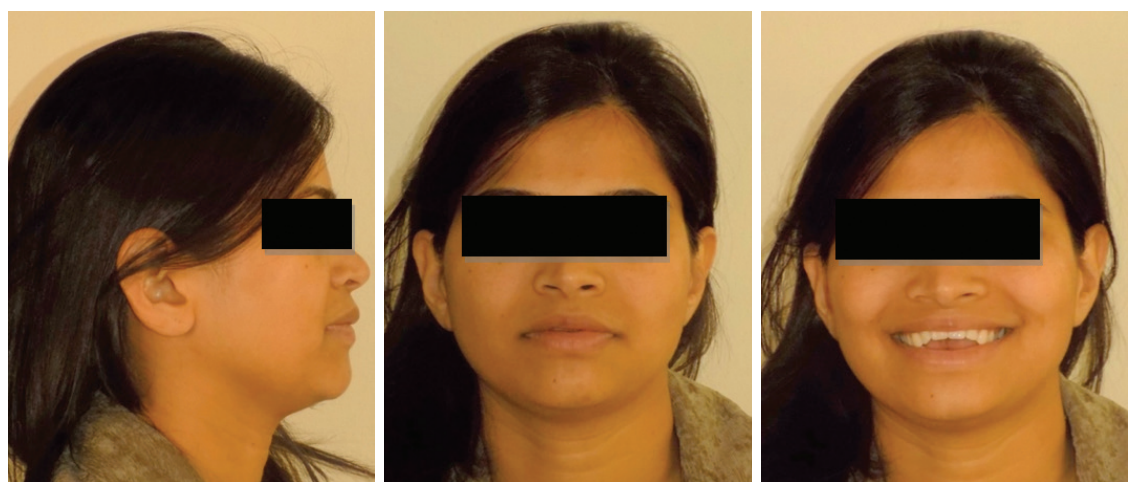

Figure 1: Pre-treatment Extra-oral photograph
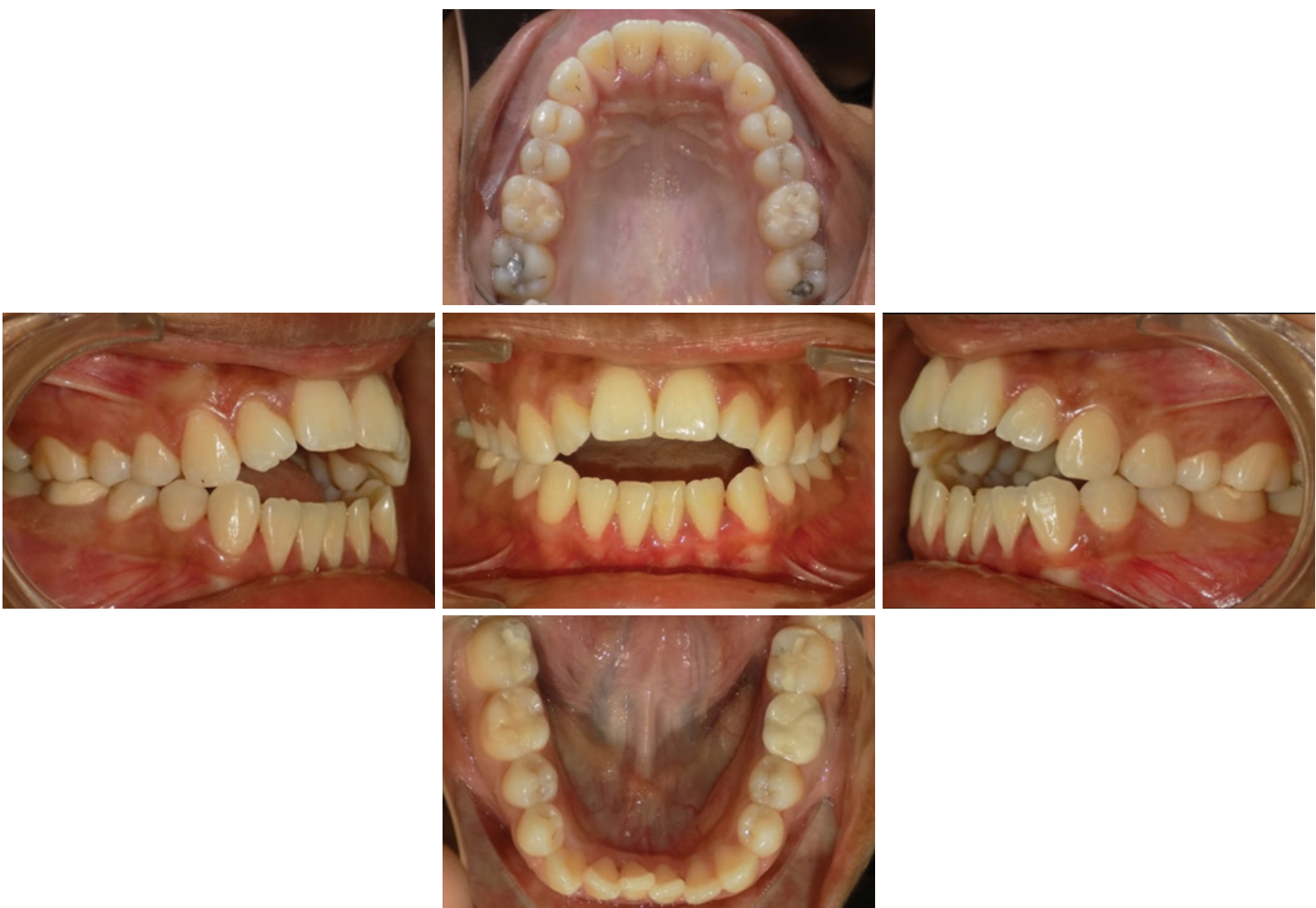

Figure 2: Pre-treatment intra-oral photographs
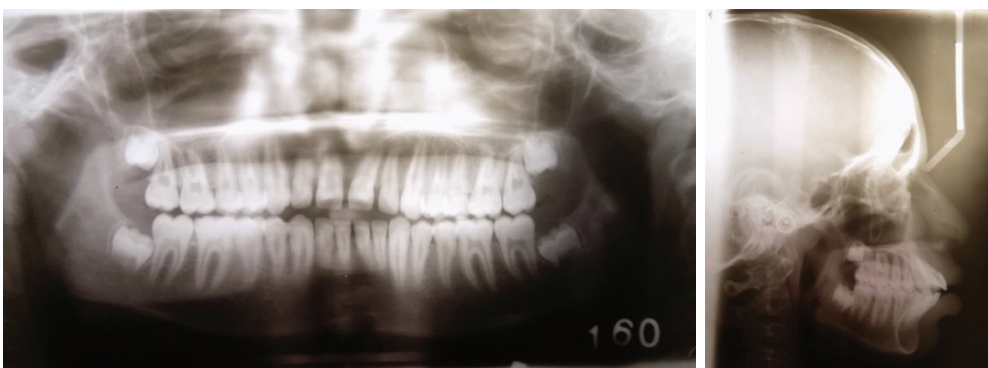

Figure 3: Pre-treatment lateral cephalogram and orthopantomogram 
Table 1: Represents cephalometric analysis

\begin{tabular}{|c|c|c|}
\hline MEASUREMENT & PRE TREATMENT & POST TREATMENT \\
\hline SNA & 87 & 83 \\
\hline SNB & 81 & 2 \\
\hline ANB & 6 & 60 \\
\hline S-GN-FH & 63 & 30 \\
\hline FMA & 35 & 65 \\
\hline ANS-Me & 68 & 2 \\
\hline Over jet & 0 & 2 \\
\hline Overbite & -10 & 112 \\
\hline Ulto Sn & 113 & 94 \\
\hline Impa & 88 & 28 \\
\hline U1-NF(mm) & 23 & 20 \\
\hline U6-NF $(\mathrm{mm})$ & 22 & 40 \\
\hline L1-MP $(\mathrm{mm})$ & 42 & 32 \\
\hline L6-MP $(\mathrm{mm})$ & 37 & \\
\hline
\end{tabular}

\section{Treatment Objectives}

Our treatment objectives included improving the patient's smile aesthetics and facial profile along with a harmonious occlusion and interception of chronic digit sucking habit.

This included:

- Creating a normal overbite and over jet relationship

- Improved facial profile

- Correct proclination of upper and lower anterior

- Correct lower crowding

\section{Treatment plan}

In this case treatment objectives were to level and align the arches with correction of convex profile and interception and correction of thumb sucking habit of the patient. The treatment planned was non extraction treatment plan with habit breaking appliance i.e. tongue crib with palatal button was placed (Fig 4) .A transpalatal arch appliance was placed between the first molars to compensate for the buccal crown torque

\section{Retention protocol}

Fixed permanent retainers were delivered. (Fig. 8).

\section{Treatment progress}

The appliance used for the patient was $0.022 \times 0.028$ " pre adjusted edgewise [MBT] fixed appliance. The treatment initiated with the incorporation of habit breaking appliance i.e. tongue crib therapy (Fig.4) for 4 months along with initial levelling and aligning with a 0.014" NiTi arch wire in the upper and lower arch.
The following sequence of wires was used: $0.016 " \mathrm{NiTi}$ $0.016 \times 0.022$ " NiTi, $0.017 \times 0.025$ " NiTi, $0.019 \times 0.025 "$ SS and a $0.021 \times 0.025$ " SS. Box Elastics were given to the patient in the anterior region in the second stage of orthodontic treatment. After space closure was completed, fixed retainers were delivered to the patient. Finishing and detailing were performed and then appliance was deboned.

\section{Post-Treatment Assessment}

Post-treatment records show a significant extrusion of the upper and lower anterior. The proclination of the upper and lower anterior was corrected and an ideal over jet, overbite and coincident midlines were achieved (Fig. 5 after space closure intra-oral photos) a remarkable improvement in the patient's aesthetics. There is a remarkable improvement in the patient's soft tissue profile (Fig. 6 after space closure-extra oral photographs). Pre-treatment and post-treatment cephalometric changes can be appreciated in Table 1. Figures 7 and 8 show post treatment photographs and Figure 9 shows post-treatment radiographs and Figure 10 shows superimposition of pre- and post-treatment radiographs by Bjork superimposition method. The patient's smile aesthetics and facial balance were improved at the end of treatment. The lips and chin appeared more aesthetic Overall superimposition of cephalometric tracings (pre-treatment, post treatment and one year follow up) showed extrusion of the anterior upper and lower incisors with little skeletal change and mandibular counterclockwise rotation. The post-treatment panoramic radiograph showed overall parallelism of roots. No significant root resorption was noted (Fig. 9). 

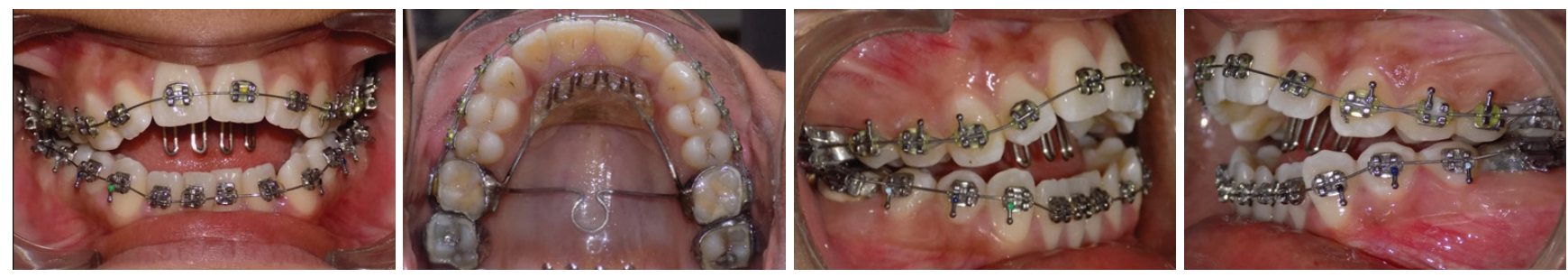

Figure 4: Habit breaking appliance
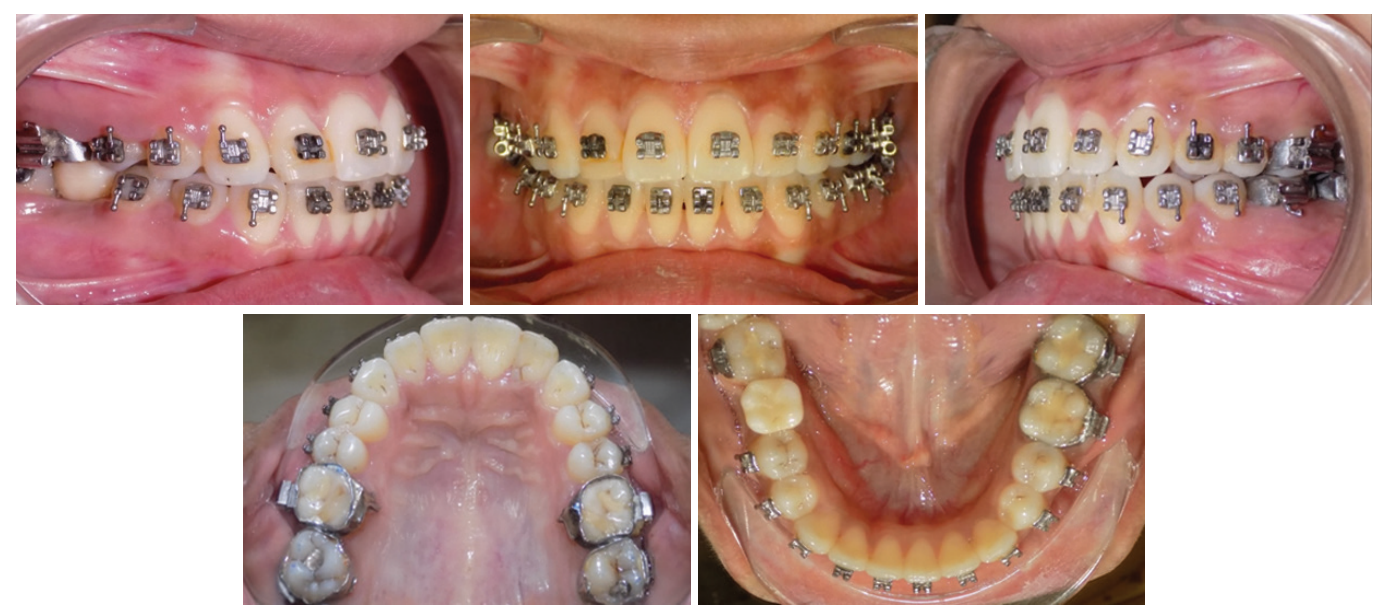

Figure 5: After space closure intra-oral photographs

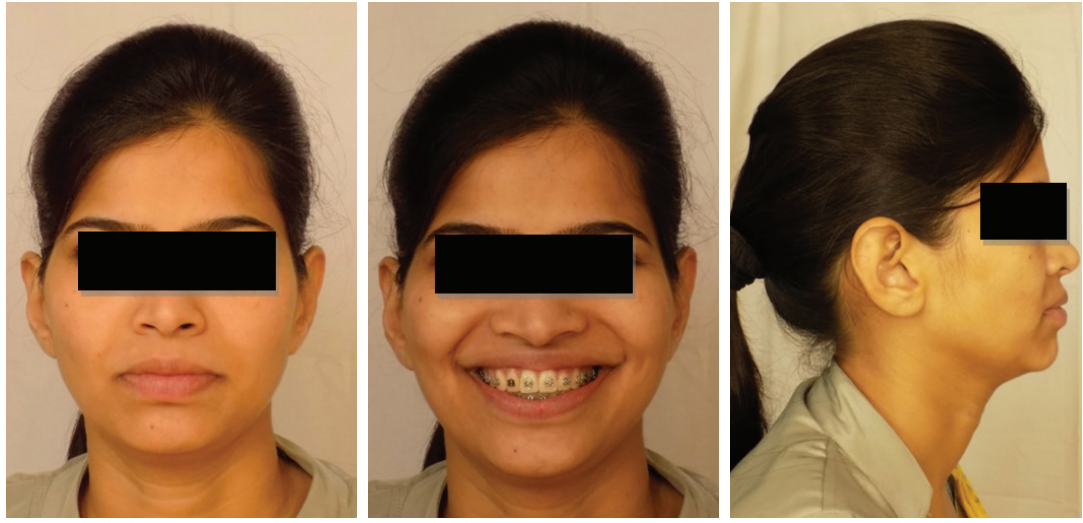

Figure 6: After space closure extra-oral photographs

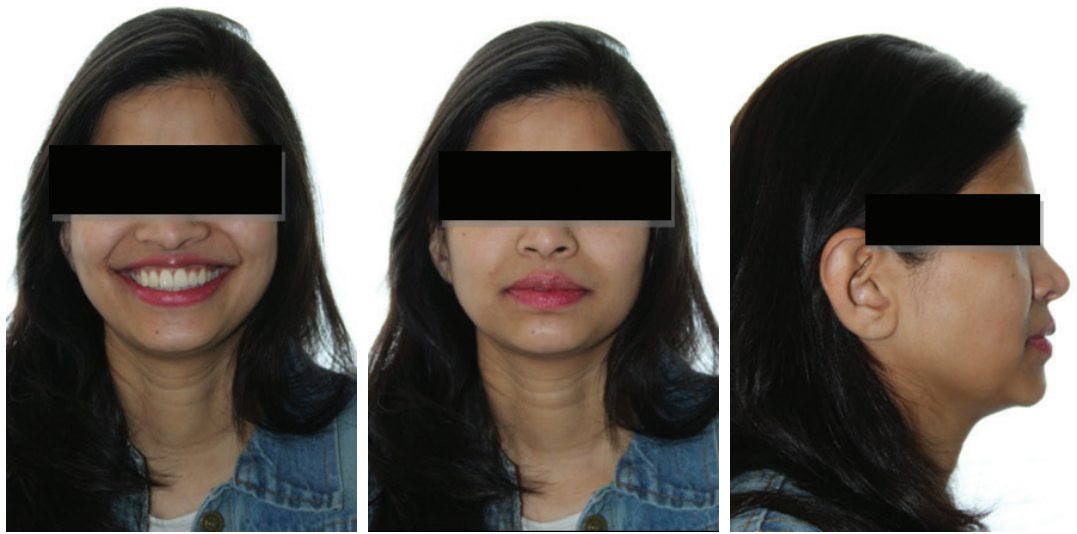

Figure 7: Extra oral photographs post debonding 

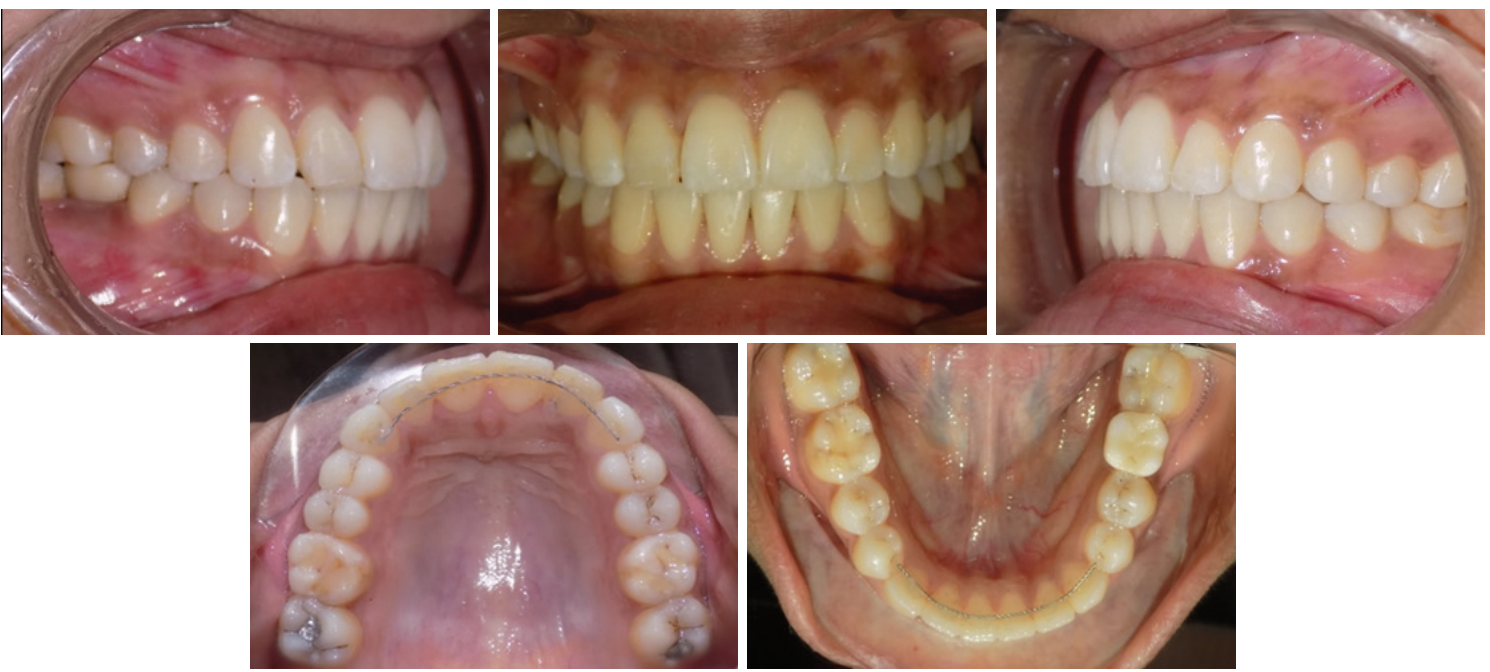

Figure 8: Intraoral photographs post debonding

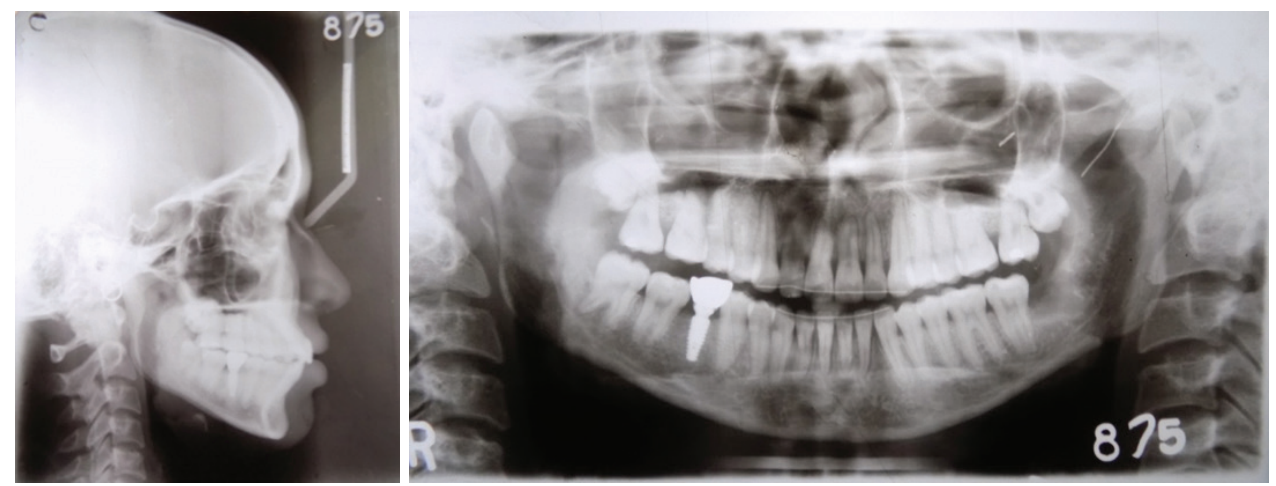

Figure 9: Post-treatment radiographs

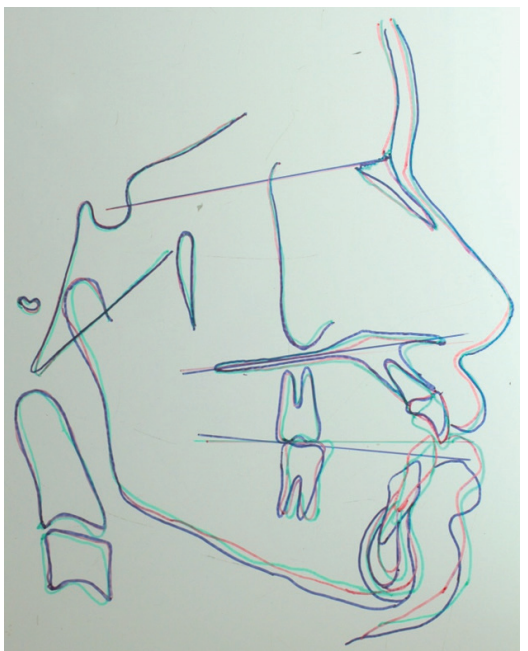

Figure 10: Superimposition by Bjork's superimposition method showing pre-treatment (-), post-treatment (-) and one year follow up(-)

\section{DISCUSSION}

The major etiologic factor that contributes to open bite is an imbalance between the tongue and the perioral musculature. ${ }^{17}$ The force of tongue or finger against the lingual surface of the incisors also cause concurrent flaring of the upper anterior teeth. ${ }^{18}$
Spontaneous interruption of a non-nutritive sucking habit before age 5 aids in the natural correction of anterior open bite, depending on the patient's facial pattern and respiratory, phonetic, and anatomical characteristics. ${ }^{10-12,14}$ This habit needs to be intervened using habit breaking appliance which includes tongue crib along with palatal button. The acrylic component 
reduced the pleasure of sucking, helping to break the habit, while the palatal grid restricted tongue pressure on the anterior teeth, favouring correct tongue posture and making the pacifier habit difficult to practice. Elimination of habits, weak musculature, and respiratory obstructions might decrease the rate and severity of relapse. An effective method of retention is recommended to improve the long term stability of open bite malocclusion. Fixed retention is the retention protocol which needs to be followed to prevent relapse in such cases. Baek et al $^{19}$ advocated using an active retainer, which consists of clear vacuumformed retainer with buttons attached to buccal or palatal side of maxillary molar. Positioners can be routinely suggested during the retention phase. Masticatory muscle exercise involving a chewing gum or soft bite wafer during retention might aid in retaining the obtaining result. ${ }^{20}$

\section{CONCLUSION}

The problem of long face and open bite is multifactorial. Diagnosis should be determined in context of the skeletal structure and the dental structure. Anterior open bite accompanied by a normal lower face height can be treated successfully using fixed appliance therapy if the aetiology can be identified as a habit or obvious environmental influence. Treatment to correct malocclusion relies on molar vertical control or extrusion of anterior segments. Long term stability remains the major challenge regardless of therapy chosen.

\section{REFERENCES}

1. Ellis III E, MCNamara Jr JA. Components of adult Class III open-bite malocclusion. American Journal of orthodontics. 1984 Oct 1;86(4):27790

2. Sassouni V, Nanda S. Analysis of dentofacial vertical proportions. American Journal of Orthodontics. 1964 Nov 1;50(11):801-23.

3. Schudy FF. Cant of the occlusal plane and axial inclinations of teeth. The Angle Orthodontist. 1963 Apr;33(2):69-82.

4. Sassouni V. A classification of skeletal facial types. American journal of orthodontics. 1969 Feb 1;55(2):109-23.

5. Parker JH. The interception of the open bite in the early growth period. The Angle orthodontist. 1971 Jan;41(1):24-44.

6. Viazis AD, Viazis E, Pagonis TC. The Fallacy of Tongue Thrust and Non-Surgical Treatment of a Severe Anterior Open Bite. J Dent Health Oral Disord Ther. 2016;4(4):00120.

7. Proffit WR, Fields HW. Occlusal forces in normal-and long-face children. Journal of dental research. 1983 May;62(5):571-4.

8. Andrianopoulos MV, Hanson ML. Tongue-thrust and the stability of overjet correction. The Angle Orthodontist. 1987 Apr;57(2):121-35.

9. Xu K, Zeng J, XU T. Effect of an intraoral appliance on tongue pressure measured by force exerted during swallowing. American Journal of orthodontics and dentofacial orthopedics. 2016 Jan 1;149(1):55-61.

10. Phelan, A.; Franchi, L.; Baccetti, T.; Darendeliler, M.A.; and McNamara, J.A. Jr.: Longitudinal growth changes in subjects with open-bite tendency: A retrospective study, Am. J. Orthod. 145:28-35, 2014.

11. Vasconcelos FM, Massoni AC, Heimer MV, Ferreira AM, Katz CR, Rosenblatt A. Non-nutritive sucking habits, anterior open bite and associated factors in Brazilian children aged 30-59 months. Brazilian dental journal. 201 1;22(2):140-5.

12. Heimer MV, Tornisiello Katz CR, Rosenblatt A. Non-nutritive sucking habits, dental malocclusions, and facial morphology in Brazilian children: a longitudinal study. The European Journal of Orthodontics. 2008 Dec 1;30(6):580-5.

13. Artese A, Drummond S, Nascimento J, Artese F. Criteria for diagnosing and treating anterior open bite with stability. Dental Press J Orthod. 2011 May; 16(3):136-61.

14. Sousa RV, Ribeiro GL, Firmino RT, Martins CC, Granville-Garcia AF, Paiva SM. Prevalence and associated factors for the development of anterior open bite and posterior crossbite in the primary dentition. Brazilian dental journal. 2014;25(4):336-42.

15. Borrie FR, Bearn DR, Innes NP, Iheozor-Ejiofor Z. Interventions for the cessation of non-nutritive sucking habits in children. Cochrane Database of Systematic Reviews. 2015(3).

16. Leite JS, Matiussi LB, Salem AC, Provenzano MG, Ramos AL. Effects of palatal crib and bonded spurs in early treatment of anterior open bite: A prospective randomized clinical study. The Angle Orthodontist. 2016 Sep;86(5):734-9.

17. Winders RV. Forces exerted on the dentition by the perioral and lingual musculature during swallowing. The Angle Orthodontist. 1958 Oct;28(4):226-35.

18. Larson E. Dummy and finger sucking habits with special attention to their significance for facial growth and occlusion4. Effect on facial growth and occlusion. Sven TandlakTidskr.1972;65(12):605-634.

19. Baek MS, Choi YJ, Yu HS, Lee KJ, Kwak J, Park YC. Long-term stability of anterior open-bite treatment by intrusion of maxillary posterior teeth. American Journal of Orthodontics and Dentofacial Orthopedics. 2010 Oct 1;138(4):396-e1.

20. Masumoto N, Yamaguchi K, Fujimoto S. Daily chewing gum exercise for stabilizing the vertical occlusion. Journal of oral rehabilitation. 2009 Dec;36(12):857-63. 